\title{
Is mechanical stress an important pathogenic factor in hidradenitis suppurativa?
}

\section{Kim de Winter, Hessel H. van der Zee and Errol P. Prens}

Department of Dermatology, Erasmus MC, University Medical Center, Rotterdam, The Netherlands

Correspondence: Hessel H. van der Zee, MD, Erasmus MC, University Medical Center, Departments of Immunology and Dermatology, Room Ee 853, Dr Molewaterplein 50, 3015 GE Rotterdam, The Netherlands, Tel.: +31-10-7043430, Fax: +31-10-7044731, e-mail h.vanderzee@erasmusmc.nl

Abstract: Hidradenitis suppurativa (HS) is a chronic of recurrent, inflammatory, follicular disease that usually presents after puberty with painful deep-seated, inflamed lesions in the inverse skin areas of the body. It has been hypothesized that mechanical pressure or friction is a risk factor for HS. We describe the case of a man with a lower leg amputation who presented HS-like lesions on his leg stump after wearing a leg prosthesis. Although pilonidal sinuslike disease could not be excluded, we diagnosed him with
HS-like lesions, induced by prosthesis-related friction. We argue that this case supports the concept that mechanical friction and a warm humid microclimate by occlusion contribute to HS development.

Key words: Acne inversa - friction - hidradenitis suppurativa - leg stump - prosthesis

Accepted for publication 4 January 2012

\section{Introduction}

Hidradenitis suppurativa (HS) is a chronic of recurrent, inflammatory, debilitating follicular disease that usually presents after puberty with painful deep-seated, inflamed lesions in the inverse skin areas of the body with a prevalence of $1-4 \%$ (1-3). The development of fistulas is an important hallmark of severe-stage HS. Clinically, HS resembles infection, but often no bacteria are cultured $(1,2)$. Putative pathogenic factors include, genetic predisposition (3), smoking (4), obesity and alterations in host defense mechanisms (5). Currently, the proposed primary mechanism in HS is hyperkeratinization of the follicular infundibulum resulting in follicular occlusion $(5,6)$ followed by an inflammatory reaction when the dilated follicles rupture and keratin fibres and epithelial strands are spilled into the dermis (7). Treatment of HS is challenging and includes topical and/or systemic antibiotics, immunosuppressive drugs as well as surgery (5). Because the predilection sites of HS (8) are rich in apocrine sweat glands, HS was initially considered an inflammatory process originating from these glands (5). However, histological examination of HS lesions demonstrated apocrinitis only in a minority of cases, suggesting that apocrinitis is a secondary event (9). Why HS prefers the inverse skin areas is unknown. In this report, we describe a case of persistent HS-like lesions with fistulas on a leg amputation stump, suggesting that mechanical friction and a warm humid microclimate by occlusion are extrinsic pathogenic factors for HS development. Case

A 44-year-old man presented recurrent, inflammatory deep-seated nodules, abscesses and fistulas on the amputation stump of his left leg. Five years earlier, he had been involved in a serious motorcycle accident, resulting in amputation of his left lower leg, and brain trauma, resulting in pituitary dysfunction. Since the amputation, he used a lower leg prosthesis. Because of his pituitary dysfunction, he used: somatoprine $0.2 \mathrm{mg}$ daily, testosterone gel $50 \mathrm{mg}$ daily; in addition, he used metformine $500 \mathrm{mg}$ daily for diabetes type 2, clobazam $10 \mathrm{mg}$ BID and budesonide/formoterol $200 / 6 \mu \mathrm{g}$ for asthma. The inflammatory lesions had previously been treated by several courses of oral antibiotics, despite cultures showing only commensal skin flora, and several times by surgical lancing. Furthermore, his prosthesis was frequently adjusted for an optimal fit. We observed multiple draining fistulas, painful nodules, abscesses, follicular keratotic papules and some pustules, especially at pressure points (Fig. 1-3). He was overweight with a BMI of 31.3 and was a smoker. He never had HS lesions in inverse skin areas and did not have a family history of HS.

We diagnosed him with HS-like lesions induced by prosthesisrelated friction and occlusion. The follicular keratosis was treated with 5\% resorcinol cream, also after deroofing. The fistulas and abscesses were surgically removed using the deroofing technique under general anaesthesia (Fig. 4). No hairs were observed in the fistulas. Up to 7 months of follow-up, the inflammatory lesions did not reoccur.

\section{Discussion}

Lower limb amputees can experience several skin problems of their stump (10). These problems comprise acroangiodermatitis, allergic contact dermatitis, bullous diseases, epidermal hyperplasia including epidermoid cysts and hyperkeratotic papules, hyperhidrosis, infections and ulcers (11). Most of these disorders are related to shear forces and increased humidity induced by the prosthesis (11). Mechanical friction can lead to epidermal hyperplasia, epidermoid cysts and hyperkeratotic papules and acne mechanica at the site of the leg stump $(11,12)$. However, deep abscessing inflammation with fistula formation has to our knowledge not been described earlier. The lesions in this case clinically resembled HS. Other characteristics in support or appropriate by a HS-like disease include a lesional exudate that yielded only commensal skin flora, no response to antibiotic treatment and the fact that the patient was a heavy cigarette smoker and overweight. Interestingly our patient never had recurrent HS-like lesions in the axilla or groin, nor a family history of HS, which is present in $40 \%$ of HS cases (8).

No hairs were observed in the fistulas. However, pilonidal sinus can formally not be excluded, although this disease is usually restricted to one inflamed nodule or abscess with fistula formation, rather than multiple nodules and sinuses. Moreover, we argue that some presumed cases of pilonidal sinus are actually HS. Clinically the diseases are often identical. This is probably because both are the result of an exaggerated inflammatory response to foreign 


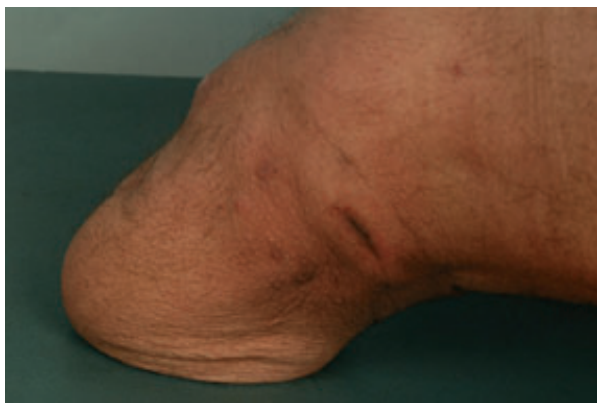

Figure 1. Left lower leg stump with fistulas and follicular keratotic papules.

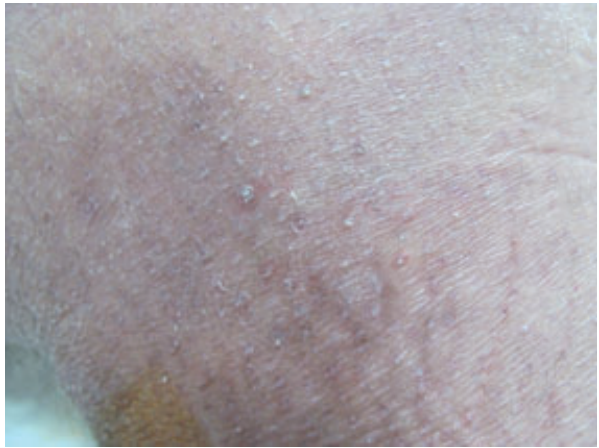

Figure 2. Detail of follicular keratotic papules on the medial side of the knee.

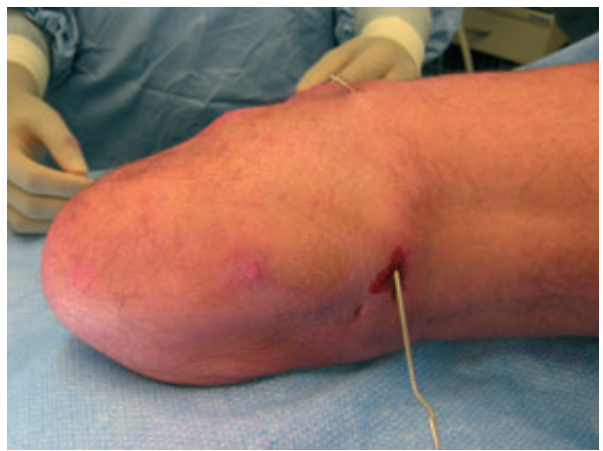

Figure 3. Probed fistula.

material, hairs in pilonidal sinus and keratin remnants in HS. But, we argue that the pathogenesis prior to the inflammation differs in both diseases, mechanical perforation of the epidermis by hairs in pilonidal sinus and follicular occlusion followed by rupture in HS.

The exact pathogenic mechanism by which mechanical friction contributes to HS development is unknown. Friction may promote HS in a twofold manner. First, it may promote follicular

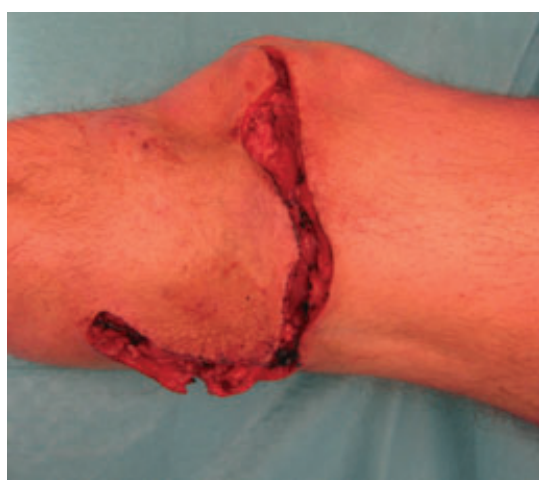

Figure 4. Extent of fistulas after surgical deroofing and slight approximation by sutures.

keratosis, similar to acne mechanica. Second, it may promote rupture of fragile dilated follicles as postulated in a case of an infant with a naevus comedonicus located in the groin, in which HS-like lesions developed only after the child started to move around (13).

A leg prosthesis creates a occlusive warm humid climate, favourable for bacterial growth, and it has been postulated that skin commensal microbiota trigger an immune response that may initiate HS. We hypothesize that these factors favoured the HS-like lesions in our case. This concept, furthermore, provides a rationale why HS is more common in the obese, where HS prefers the increased and extended abdominal skinfolds and the inner thighs, all characterized by enhanced mechanical friction, occlusion and by a warm and humid microclimate.

We treated the patient with the surgical deroofing technique. With this technique, the 'roof of an abscess, cyst or sinus tract is electro-surgically removed, and probe is used to explore fistulas (14).

In summary, we present a patient with inflammatory lesions, resembling HS, at an ectopic location free of apocrine sweat glands and probably induced by a leg prosthesis. We argue that predilection sites of HS are not characterized by the presence of apocrine sweat glands but instead by mechanical friction and a warm humid, occlusive microclimate favourable to bacteria.

\section{Acknowledgments}

Kim de Winter: Saw patient at first visit and wrote paper; Hessel H. van der Zee: Supervised patient visits and wrote paper as well the resubmission; Errol P. Prens: Supervised patient visits and manuscript and performed deroofing on the patient.

\section{Funding}

This study was not externally funded.

\section{Conflicts of interest}

The authors have declared no conflicting interests.

\section{References}

1 Jemec G B, Faber M, Gutschik E et al. Dermatology 1996: 193: 203-206.

2 Lapins J, Jarstrand C, Emtestam L. Br J Dermato 1999: 140: 90-95.

3 Al-Ali $F$ M, Ratnamala U, Mehta $T$ Y et al. Exp Dermatol 2010: 19: 851-853.

4 Lukowsky A, Sterry W, Schneider-Burrus S. Exp Dermatol 2010: 19: 541-542.

5 Alikhan A, Lynch P J, Eisen D B. J Am Acad Dermatol 2009: 60: 539-561.
6 von Laffert M, Helmbold P, Wohlrab J et al. Exp Dermatol 2010: 19: 533-537.

7 van der Zee $\mathrm{H} \mathrm{H}$, de Ruiter L, Boer J et al. $\mathrm{Br} J$ Dermatol 2012: 166: 98-106.

8 von der Werth J M, Williams H C. J Eur Acad Dermatol Venereol 2000: 14: 389-392.

9 Jemec G B, Hansen U. J Am Acad Dermatol 1996: 34: 994-999.

10 Meulenbelt $\mathrm{H} \mathrm{E}$, Geertzen J H, Jonkman M F et al. Arch Phys Med Rehabil 2009: 90: 74-81.
11 Meulenbelt H E, Geertzen J H, Dijkstra P U et al. J Eur Acad Dermatol Venereol 2007: 21: 147155.

12 Ibbotson S H, Simpson N B, Fyfe N C et al. Br J Dermatol 1994: 130: 770-772.

13 Dufour D N, Bryld L E, Jemec G B. Dermatology 2010: 220: 323-325

14 van der Zee H H, Prens E P, Boer J. J Am Acad Dermatol 2010: 63: 475-480. 